\title{
Classificação analítico-sintética: reflexões teóricas e aplicações
}

\section{Analytical-synthetic classification: theoretical reflections and applications}

Lígia Maria Arruda CAFÉ'

Aline BRATFISCH${ }^{2}$

\section{RESUMO}

Trata de reflexões teóricas e aplicações práticas em modelos de organização do conhecimento do tipo analíticosintético, disponíveis na literatura. Utiliza como fonte de coleta as bases de dados Wilson Library Literature and Information Science Full Text e Library and Information Science Abstracts (LISA), bem como as referências bibliográficas presentes nos documentos identificados nessas fontes. Define o período 1995 a 2005, priorizando tanto publicações nacionais como publicações estrangeiras.

Palavras-chave: análise facetada; classificação facetada.

\section{A B S T R A C T}

Research on theoretical reflections and practical applications in analytical-synthetic knowledge organizational models available in literature. It uses as sources the database of Wilson Library Literature and Information Science Full Text and Library and Information Science Abstracts (LISA), as well as the bibliographical references of the documents identified in said sources. The analysis covers Brazilian and foreign publications in the period of 1995-2005.

Keywords: faceted analysis; faceted classification.

\section{INTRODUÇÃ O}

No contexto moderno das bibliotecas digitais e virtuais, a interoperabilidade entre os diversos repositórios é a palavra-chave para alcançar a qualidade na transferência da informação. Trata-se não apenas da adoção de padrões e métodos tecnológicos, mas igualmente da aplicação da interoperabilidade semântica. Essa última contribui para o aumento da precisão e revocação no processo de recuperação da

\footnotetext{
1 Docente, Departamento de Ciência da Informação, Programa de Pós-Graduação em Ciência da Informação, Universidade Federal de Santa Catarina. Campus Universitário Trindade, s/n, Trindade, 88040-900, Florianópolis, SC, Brasil. Correspondência para/ Correspondence to: L.M.A. CAFÉ. E-mail: <ligia@cin.ufsc.br>.

2 Aluna do Programa de Bolsas de Iniciação Científica (PIBIC), Departamento de Ciência da Informação, Universidade Federal de Santa Catarina. Florianópolis, SC, Brasil. E-mail: <a_bratfisch@hotmail.com>.

Recebido em 12/3/2007 e aceito para publicação em $\overline{1} / 8 / 2007$.
} 
informação e no intercâmbio entre diferentes canais de informação.

Os instrumentos utilizados para atingir a interoperabilidade semântica são variados: ontologias, classificações, tesauros, entre outros. Apesar das denominações diferenciadas, eles se aproximam em determinados pontos funcionais e metodológicos. Em nível funcional, Soergel (1999) enumera vários objetivos comuns entre esses instrumentos. Destacamos:

$\checkmark$ Fornecer um mapa semântico de campos individuais do conhecimento e relação entre eles [...];

$\checkmark$ Melhorar a comunicação e o aprendizado $[\ldots]$;

$\checkmark$ Fornecer uma base conceitual para 0 planejamento de uma pesquisa de qualidade e sua implementação [...];

$\checkmark$ Apoiar a recuperação da informação: oferecer suporte baseado no conhecimento para busca pelo usuário final (menu em árvores, análise facetada guiada para um tópico de busca, navegação em mapa conceitual ou hierárquico para identificação de conceitos pertinentes para uma pesquisa, mapeamento partindo dos termos da questão do usuário e identificando descritores utilizados em uma ou mais base de dados ou expressões múltiplas da linguagem natural para busca livre em texto), oferecer busca expandida hierarquicamente, apoiar saídas bem estruturadas para resultados de busca, oferecer um instrumento para indexação (controle de vocabulário, indexação centrada no usuário ou orientada ao problema) ${ }^{3}$ (tradução nossa).
Do ponto de vista metodológico, os instrumentos de base analítico-sintéticos parecem oferecer maiores possibilidades de descrição e recuperação de conteúdos multidimensionais exigidas pelos Sistemas de Recuperação da Informação. Na verdade, essas ferramentas vêm ocupando espaço cada vez maior no âmbito das modernas teorias de representação do conhecimento. Os modelos baseados na lógica analítico-sintética superam em grande parte outros métodos como aqueles aplicados somente na elaboração de estruturas hierárquicas. Dahlberg (1978, p. 105), ao descrever o método de categorização conceitual utilizado nas classificações facetadas, ressalta suas "[...] possibilidades inúmeras de combinações entre as categorias". Os esquemas cuja organização se apóia apenas em estruturas hierárquicas, embora forneçam uma visualização do conhecimento de forma global, não oferecem alternativas precisas de associação entre as classes, além de serem bastante precários na representação de conteúdos semânticos complexos.

As pesquisas desenvolvidas pelo Classification Research Group (CRG) seguem nessa linha de pensamento, privilegiando o trabalho analítico-sintético. Suas investigações, iniciadas em 1952, relatam que

Os termos subordinados a um determinado gênero - p.ex., uma classe principal - não são todos derivados desse gênero por diferenciação, utilizando uma única característica de divisão. Eles podem ser agregados em grupos de facetas, cada um dos quais derivado do gênero por meio de uma característica diferente. De forma a mostrar adequadamente a ligação entre termos relacionados, é necessário aplicar esquemas de classificação facetada ${ }^{4}$.

3 - Provide a semantic road map to individual fields and the relationships among fields [...];

- Improve communication and learning [...];

- Provide the conceptual basis for the design of good research and implementation [...];

- Support information retrieval: provide knowledge-based support of end-user searching (menu trees, guided facet analysis of a search topic, browsing a hierarchy or concept map to identify search concepts, mapping from the user's query terms to descriptors used in one or more data-bases or to the multiple natural language expressions for free-text searching); support hierarchically expanded searching; support well-structured displays of search results; provide a tool for indexing (vocabulary control, user-centered or problem-oriented indexing).

4 The terms subsumed under a given genus - e.g., a main class - are not all derived from thar genus by differentiation using a single characteristic of division. They can be sorted into group or facets, each of which is derived from the genus by a different characteristic. In order adequately to display the linkage of related terms, it is necessary to have faceted classification schedules. 
(CLASSIFICATION RESEARCH GROUP, 1985, p. 158) (tradução nossa).

O princípio da análise facetada foi definido por S. R. Ranganathan (1892-1972), um dos maiores contribuidores da teoria da análise de assuntos. Tratase de um método sistemático consistente de agrupamento conceitual de termos pertencentes a uma mesma classe. Ranganathan (1985, p.88), determinou cinco categorias fundamentais para a interpretação de qualquer assunto: Personalidade, Matéria, Energia, Espaço e Tempo. Pesquisas mais recentes têm procurado demonstrar que a análise de um determinado assunto pode ir além dessas categorias, o que não invalida seu valor teórico e metodológico para o desenvolvimento da pesquisa sobre classificação. Na verdade, trata-se de um instrumento consolidado, cujos

Princípios de categorização implícitos na teoria [...] podem ser estendidos para a organização de qualquer conjunto de propriedades de objetos em qualquer área do conhecimento [...]. A teoria está suficientemente bem estabelecida para permitir variação na forma clássica, e o compilador da estrutura facetada não deve se sentir restrito as categorias ou regras combinatórias da ordem de citação padrão ${ }^{5}$ (Broughton, 2002) (tradução nossa).

No âmbito da construção de modelos de representação do conhecimento do tipo tesauro, encontra-se uma linha moderada que segue como ideal a construção de classificações combinando o método hierárquico com o analítico-sintético. Segundo Maple (1995),

Pesquisas recentes em recuperação da informação sugerem que construir um tesauro que mostre tanto as relações semânticas como sintáticas ${ }^{6}$ terão potencialmente grandes benefícios aos nossos usuários no futuro ${ }^{7}$ (tradução nossa).
Esse modelo híbrido reflete toda a complexidade de significações do conhecimento analisado. No eixo semântico, são interpretadas as relações permanentes de hierarquia existentes entre os conceitos. No eixo sintático, são examinadas as funções sintáticosemânticas resultantes da combinatória dos constituintes $\mathrm{do}(\mathrm{s})$ termo(s) que representa $(\mathrm{m})$ um conceito ou um assunto complexo.

É no contexto dos instrumentos de representação do conhecimento que seguem o princípio analíticosintético que se delimita este artigo. Seu conteúdo é resultado da primeira parte da pesquisa "Critérios lingüísticos adotados em modelos de organização do conhecimento", do Programa de Pós-graduação em Ciência da Informação da Universidade Federal de Santa Catarina. Essa parte do projeto teve como objetivo levantar os critérios lingüísticos adotados por instrumentos utilizados na organização de conhecimento para recuperação da informação. Observou-se que a maioria das publicações encontradas não tratava de forma explícita dos critérios lingüísticos. No entanto, por meio da descrição de experiências teóricas e aplicações práticas, deixavam registradas algumas indicações a esse respeito. Após a descrição da metodologia adotada, apresentamos um resumo de cada texto identificado, trazendo uma síntese das questões teóricas e metodológicas nele descritas.

\section{Metodologia e instrumento de registro dos dados}

O levantamento dos dados desta pesquisa adota o método de análise de conteúdo definido por Bardin (2003). Segue, portanto, as três fases determinadas pela autora:

1. Pré-análise;

2. Exploração do material;

\footnotetext{
${ }^{5}$ The principles of categorization implicit in [...] theory can be extended to organize any set of properties of objects in any domain [...]. The theory is sufficiently well established to allow variation in the classical form, and the compiler of a faceted structure need not feel restricted to the categories and combinatorial rules of standard citation order.

6 Para Maple, as relações semânticas dizem respeito as relações permanentes (de equivalência, hierárquicas e associativas), enquanto que as relações sintáticas relacionam-se a combinação dos conceitos para representar os assuntos dos documentos.

${ }^{7}$ Current research in information retrieval suggest that constructing a thesaurus that sows both semantic and syntactic relationship will have potentially great benefits to our users in the future.
} 
3. Tratamento dos resultados, inferências e interpretação.

$\mathrm{Na}$ fase de pré-análise, foram coletados documentos (principalmente artigos e relatórios de pesquisa) que tratavam do assunto pesquisado de maneira a constituir um corpus de pesquisa.

Aplicamos os princípios de exaustividade, representatividade, homogeneidade e pertinência conceituados por Bardin (2003, p.127-128) para selecionar os documentos identificados em cada busca. Dessa forma, procuramos coletar a grande maioria do material disponível (exaustividade), observando sua representatividade em relação ao universo pesquisado, descartando publicações muito específicas que fugiam aos critérios de escolha (homogeneidade), assim como retendo apenas o material relevante no que concerne ao assunto da pesquisa (pertinência).

As buscas foram realizadas nas bases de dados Wilson Library Literature and Information Science Full Text e Library and Information Science Abstracts (LISA) por meio dos descritores "Faceted classification", "Faceted analysis" e "Análise Facetada" e "Classificação Facetada". No intuito de completar o levantamento, identificamos publicações pertinentes nas listas de referências de cada documento recuperado. Delimitamos o levantamento aos últimos 10 anos (1995 a 2005).

A base de dados Wilson Library Literature and Information Science Full Text consiste em uma coleção de periódicos, teses e livros com textos completos ou apenas resumos, disponível no Portal de Pesquisa, cobrindo, entre outras áreas, a de Ciência da Informação.

A base de dados LISA constitui-se de um repertório internacional de resumos na área de Ciência da Informação que cobre 440 periódicos de mais de 68 países em mais de 20 línguas.

Diversos documentos nacionais foram encontrados nessas bases, no entanto pode-se dizer que as publicações internacionais tiveram maior peso.

$\mathrm{Na}$ fase de exploração do material, foram determinadas, por meio de discussões acerca do que seria relevante a ser registrado dos documentos coletados, as categorias para registro dos dados do corpus, seguindo os seguintes critérios expostos por Bardin (2003, p. 153-154):
1. Exclusão mútua;

2. Homogeneidade;

3. Pertinência;

4. Objetividade e fidelidade;

5. Produtividade.

Assim, identificamos as seguintes categorias registradas como campos em uma base de dados Access:

1. Referência bibliográfica do documento: foram incluídos os dados de autor, título, editor, data de publicação, volume, página, mês, ano, e outros dados bibliográficos complementares.

2. Indicação do tipo de conteúdo do documento: os tipos de documentos incluídos na base de dados eram classificados como teórico ou aplicado. Os documentos que refletiam sobre a teoria da classificação facetada foram classificados como teóricos. Os documentos que descreviam um tipo de aplicação, como, por exemplo, o método de organização de um tesauro facetado utilizado em uma rede de televisão ou em portais do governo, eram considerados aplicados.

3. Resumo do conteúdo do documento: foram registrados os elementos de entendimento geral do artigo, dando enfoque às partes em que a análise facetada era descrita.

4. Área de aplicação: foram registrados os tipos de aplicação da classificação facetada. Por exemplo, cita-se uma aplicação na organização de portais.

5. Área do Conhecimento: foram registradas as áreas de conhecimento onde a classificação facetada estava sendo aplicada ou refletida teoricamente.

6. Critério utilizado: foram registrados os critérios lingüísticos (morfológico, sintático ou semântico). Como exemplo, há a utilização de um critério semântico em uma aplicação de classificação facetada.

7. Indicação se critério lingüístico ou não: foi registrada a indicação de quando o critério utilizado era lingüístico ou não.

8. Observação: foram registradas informações relevantes que não estavam previstas nos outros campos. 
A fase de tratamento dos resultados, objeto deste artigo, encontra-se dividida em duas partes. A primeira refere-se aos documentos que relatam experiências de cunho teórico, sendo a segunda parte relativa às aplicações práticas cuja base é a classificação facetada. No caso das publicações teóricas, os documentos apresentavam reflexões sobre a teoria da classificação facetada, enquanto as aplicações descreviam um tipo de utilização de instrumentos facetados em organizações como redes de televisão ou em portais do governo, entre outros. Passamos então a descrever o conteúdo dos dados analisados.

\section{Experiências teóricas em classificação analítico-sintética}

No campo das reflexões teóricas, trabalhos que abordam normas e padrões de desenvolvimento de tesauros mostram lacunas no que diz a respeito à utilização de métodos de análise facetada na construção desses instrumentos. Isto é o que procura comprovar o artigo de Spiteri (1997), onde a autora expõe um estudo sobre as normas ISO $2788^{\circ}$, ANSI/NISO Z39. $19^{9}$ e BS $5723^{10}$, concluindo que essa carência metodológica traz como conseqüência a falta de consenso entre aqueles que planejam os tesauros, quanto à aplicação dos princípios analítico-sintéticos em tesauros. No argumentar da autora, ainda que somente os trabalhos de Ranganathan e do Classification Research Group (CRG) possam ser considerados como fontes de utilização correta da análise facetada, não se pode dizer que esses sejam totalmente apropriados às questões de um tesauro. Apresenta para essa afirmação duas justificativas. Em primeiro lugar, os princípios definidos por Ranganathan e pelo CRG diferem profundamente na definição e ordem das categorias fundamentais. Em segundo lugar, foram pensados tendo em vista a elaboração de classificações bibliográficas, as quais oferecem meios de descrição genérica de documentos, o que diverge da representação específica almejada pelos tesauros. Essas constatações, conclui Spiteri (1997, p.24), significam que "[...] não existe nenhum modelo ideal de análise facetada que possa ser aplicado a tesauros facetados"11 (tradução nossa).

Preocupada com a questão da aprendizagem, Spiteri (1998), em um trabalho posterior, apresenta um modelo simplificado cujo conteúdo sintetiza os princípios da análise facetada desenvolvidos por Ranganathan e CRG. Segundo a autora, esse documento objetiva, em primeiro lugar, auxiliar o ensino na área. Em segundo plano, poderá também servir a elaboradores de tesauros facetados, assim como ser um conjunto de critérios de validação da integridade estrutural de sistemas facetados. O trabalho oferece uma metodologia dividida em três partes: a) exame de cada princípio postulado por Ranganathan, indicando redundâncias e contradições, quando for o caso, b) comparação entre os princípios de Ranganathan e CRG, apontando convergências e divergências, c) consolidação do modelo simplificado constituído dos princípios convergentes e de sugestões para resolução de divergências. À guisa de conclusão, a autora relata que o modelo consolidado parece privilegiar de certo modo alguns aspectos elaborados pelo CRG, particularmente na área da escolha das categorias fundamentais e ordem de citação das facetas. A razão apontada é o fato de, aparentemente, a teoria de o CRG apresentar uma maior flexibilidade na resolução dessas questões.

Em outro artigo, Spiteri (1999) compara a aplicação dos princípios da análise facetada em 14 tesauros facetados publicados desde 1980, avaliando quais desses princípios são utilizados e de que forma. A autora chega às seguintes conclusões:

a) os tesauros não compartilham os mesmos conceitos do que vem a ser uma faceta,

b) alguns tesauros utilizam métodos mistos que envolvem tanto a classificação enumerativa quanto a facetada,

8 INTERNATIONAL STANDARD ORGANIZATION. ISO 2788: documentation: guidelines for the establishment and development of monolingual thesauri. [S.I.], 1986.

9 NATIONAL INFORMATION STANDARDS ORGANIZATION. ANSI/NISO Z39.19: Guidelines for the construction, format, and management of monolingual thesauri. Bethesda: 1994.

10 BRITISH STANDARDS INSTITUTION. BS 5723: Bristish standard guide to establishment and development of monolingual thesauri. Londres: 1987.

$11[\ldots]$ there is no one underlying model of facet analysis that can be applied to faceted thesauri. 
c) as facetas não são homogêneas e mutuamente exclusivas,

d) utilização do princípio de síntese em apenas $50 \%$ dos tesauros,

e) falta de consistência na ordem de citação.

Nessa mesma perspectiva da problemática metodológica de construção de instrumentos de tratamento da informação, Hiðrland (2002) ressalta a pouca cientificidade dos métodos de elaboração de classificações. Defende que, pelo fato de serem consideradas como padrões, as classificações não podem pretender ter status científico. Mesmo que o autor se refira aos estudos de Ranganathan e do CRG como partes importantes da literatura em Ciência da Informação - assim como suas aplicações na Classificação de Bliss (segunda edição) e na Classificação Decimal Universal -, ele aponta quatro problemas básicos com relação à abordagem analíticosintética: a) falta de métodos empíricos explícitos, b) falta de atualização metodológica, c) falta de comparação com outros métodos e d) falta de reconhecimento formal na área da Ciência da Informação. Hjorland discorre sobre cada um deles aprofundando seu pensamento. $\bigcirc$ artigo, cujo objetivo é discutir o estado-da-arte das metodologias de construção de esquemas classificatórios, parte da classificação até as ontologias em defesa de uma visão mais científica dos instrumentos de tratamento e recuperação da informação. Ao final, apresenta tendências inovadoras no campo da investigação sobre modelos de classificação. Essas tendências incluem propostas metodológicas com base em paradigmas, ou seja, segundo diferentes formas de enxergar o mundo.

Em busca de princípios fundamentais que apóiem o processo de modelização de domínios de conhecimento, Campos (2004) apresenta uma análise comparativa de teorias desenvolvidas para esse fim pela Ciência da Informação, Terminologia e Ciência da Computação. A Teoria da Classificação Facetada, juntamente com a Teoria do Conceito de Ingetraut Dahlberg, constituem aquelas escolhidas da Ciência da Informação, uma vez que abordam a representação de sistemas de conceitos. $\bigcirc$ método comparativo utiliza 4 pontos de partida: o método de raciocínio, o objeto de representação, as relações entre os conceitos e as formas de representação gráfica.
Mills (2004) expõe o importante papel da classificação facetada na recuperação da informação. Concentra sua explanação na estruturação da divisão lógica desse tipo de classificação, detalhando passo a passo o processo de análise em facetas.

Em um contexto de cunho conceitual, Maniez (1999) tece comentários sobre o uso nem sempre preciso do termo "faceta" por especialistas em Ciência da Informação. Por ser um empréstimo da língua comum, essa palavra, plena de significações, adquire o status de termo quando se insere no campo terminológico das classificações. No entanto, muitas vezes, como refere o autor, até mesmo especialistas renomados confundem o termo com a palavra. Após relatar que o conceito do termo foi originalmente determinado por Ranganathan, Maniez (1999) explora a aplicação das facetas nos eixos paradigmáticos e sintagmáticos propostos pela Lingüística e conclui que Ranganathan pretendia encontrar a sintaxe absoluta na descrição dos assuntos, o que é contestado por diversos autores. Segundo o autor, os estudos do CRG recusam as fórmulas universais de descrição do conteúdo e, ao contrário de Ranganathan, aplicam o método facetado em áreas específicas do conhecimento, não definindo nenhuma lista de facetas a priori. Maniez (1999) explora ainda questões relativas à aplicação do método de facetas em tesauros, mencionando suas vantagens e sintetiza os conceitos corretos e incorretos de faceta, objetivando esclarecer o bom uso do termo no domínio das classificações.

Gnoli (2004) procura demonstrar a atualidade da classificação facetada, cuja teoria, desenvolvida em meados do século 20, apresenta interessantes soluções para a busca em textos completos do mundo digital.

Em Glassel (1998), compara-se a teoria de Ranganathan e as classificações do Yahoo! (http:// www.yahoo.com), diretório de assuntos da Internet. Segundo a autora, algumas pessoas consideram o Yahoo semelhante a um tesauro, por utilizar palavras ao invés de símbolos de notação. No entanto, Glassel (1998) defende que o Yahoo! baseia seu acesso por uma hierarquia navegável por categorias, subcategorias, sub-subcategorias, combinando-as assim como o fez Ranganathan com as facetas na descrição de assuntos complexos. Sendo assim, considera-o ainda mais complexo que um tesauro nas suas habilidades de combinar categorias.

Cada termo na notação do Yahoo! contém palavras individuais que possuem significados 
próprios, que uma vez combinadas com outras palavras da seqüência, cria-se um contexto, fornecendo um significado mais completo. Dessa forma, assemelha-se a uma classificação facetada. ${ }^{12}$ (Glassel, 1998) (tradução nossa).

Ao expor uma revisão histórica da análise em faceta no contexto das pesquisas em recuperação da informação, Maple (1995) introduz o assunto definindo o significado do termo "faceta" e os conceitos relacionados ao tema. A autora relata que, apesar de, desde o século 18, vários instrumentos classificatórios já apresentarem características do que viria a ser denominado de faceta, a teoria foi organizada e descrita pela primeira vez na Classificação de Dois Pontos de Ranganathan em 1933. A partir de então, vários esquemas classificatórios aplicaram seus princípios e métodos de forma sistematizada. A teoria de Ranganathan é também discutida no início dos anos 50 pelo grupo britânico Classification Research Group, que direciona suas pesquisas as classificações em áreas especializadas do conhecimento. Dando prosseguimento ao relato histórico, Maple (1995) lembra o surgimento da indexação pós-coordenada, utilizada na recuperação da informação, cujo desenvolvimento derivou nos fundamentos para construção de tesauros, instrumentos baseados nas relações semânticas entre conceitos. Ao aprofundar sobre os tipos de relações entre conceitos, Maple (1995) descreve as duas relações possíveis: a semântica e a sintática e suas implicações na recuperação da informação, fornecendo inclusive exemplos de sistemas que as aplicam.

Após uma explanação sobre estudos na área de cruzamento entre lingüística e documentação e no domínio da representação documentária, Pereira e Bufrem (2005) fundamentam o artigo citando as reflexões de Maria Luiza Campos no que se refere aos princípios comuns presentes na Teoria do Conceito (Dahlberg), a Teoria Geral da Terminologia (Wüster) e a Teoria da Classificação Facetada (Ranganathan). As autoras acrescentam a esse levantamento uma síntese de algumas características relacionadas à análise documentária, justificando sua "relação ao trabalho intelectual do indexador em um sentido mais amplo" (Pereira; Bufrem, 2005, p.34).
Partindo do pressuposto de que o conhecimento utiliza como um de seus processos a classificação e que, assim como uma teoria, a classificação é uma conexão entre conceitos, Kwasnik (1999) analisa diversas abordagens classificatórias, entre as quais a classificação facetada. Para a autora,

[...] as classificações facetadas não são realmente uma estrutura representacional diferente, mas, pelo contrário uma abordagem diferente do processo de classificação. A noção de facetas se deve à crença de que existe mais de uma maneira de observar o mundo e que mesmo essas classificações, que são vistas como estáveis, são de fato provisórias e dinâmicas. desafio é construir classificações flexíveis e que possam acomodar novos fenômenos ${ }^{13}$ (KWASNIK, 1999, p. 14-15) (tradução nossa).

A autora ainda ressalta como as categorias fundamentais sobreviveram ao tempo e podem ser adotadas para os mais diversos tipos de objetos. Acrescenta ainda a grande aplicabilidade do método de análise proposto por Ranganathan. Mas, assim como qualquer outra forma de organização do conhecimento, a classificação facetada também possui algumas limitações em termos de representação e criação do conhecimento. Nesse sentido, a autora cita limitações com relação à dificuldade de estabilizar facetas apropriadas, à falta de relacionamentos entre facetas, e à dificuldade de visualização. Mesmo assim, ela ainda continua sendo importante por oferecer mecanismos sistemáticos de visualização do mundo, sem necessariamente depender de uma tecnologia interna específica para isto.

Leise (c2003) desenvolve seu artigo esclarecendo sobre a utilidade do método da classificação facetada na análise do texto e na elaboração de índices. $\bigcirc$ autor explica com exemplos a aplicação das facetas nessas atividades, especificando que esse método auxilia o indexador a definir quais facetas são mais apropriadas para o trabalho em determinados tipos de textos. Além

\footnotetext{
12 Each term in a Yahoo! Notation string contains individual words which have meaning on their own, but once combined with other words into a string, a context is created, providing a deeper meaning. In this way it is much like a faceted classification.

13 Faceted classification are not really a different representational structure but rather a different approach to the classification process. The notion of facets rests on the belief that there is more than one way to view the world, and that even those classifications that are viewed as stable are in fact provisional and dynamic. The challenge is to build classifications that are flexible and can accommodate new phenomena.
} 
disso, segundo o autor, as facetas pode ser adotadas na indexação como uma garantia à exaustividade e à consistência da indexação.

Em Tristão, Fachin e Alarcon (2004), os autores sintetizam conceitos relacionados à classificação facetada e tesauro. É ressaltado que os sistemas de classificação e tesauros vêm ocupando um espaço cada vez maior na organização do conhecimento em várias áreas, tanto em nível nacional como internacional, e que, devido aos avanços das tecnologias, esses esquemas estão sendo aplicados com sucesso na Web e em redes digitais. Após uma explanação da tipologia das classificações, o texto concentra-se na classificação facetada, mostrando as diferenças entre esses sistemas e os enumerativos. Apoiando-se em Broughton (2002), Tristão, Fachin e Alarcon $(2004$, p. 167) relatam que

[...] na classificação facetada a lógica interna do sistema é baseada em uma análise rigorosa do vocabulário, e os termos são classificados dentro de um conjunto-padrão de categorias funcionais. Dentro dessas categorias, várias relações semânticas são conhecidas e problemas de controle de vocabulário são endereçados. Um sistema de sintaxe provê a ordem e a combinação dos termos intra e entre facetas.

Tristão, Fachin e Alarcon (2004) discorrem também sobre as intersecções entre tesauro e as classificações em facetas e tratam da Teoria do Conceito e sua aplicação na construção das classificações. Baseando-se em Ranganathan, exploram questões sobre o relacionamento entre conceitos e sua tipologia.

Lima (2002) apresenta uma revisão de literatura sobre aplicações da Teoria da Classificação Facetada na resolução de questões relacionadas à organização conceitual em sistemas hipertextos, cujo objetivo é possibilitar ao usuário visualizar um assunto sob diferentes perspectivas. Mostra a relevância dessa base teórica que auxilia na solução da problemática de organização do conhecimento em abordagens não lineares, característica específica dos hipertextos.

\section{Aplicações práticas em classificação facetada}

No que tange às aplicações práticas da classificação facetada, os documentos analisados apresentavam uma tipologia variada. Esse fato revela a atualidade do método analítico-sintético, assim como sua flexibilidade de aplicação independente do estágio de desenvolvimento das tecnologias.

Destacamos inicialmente o trabalho de Araújo (2003) que, em uma perspectiva mais concreta, propõe a elaboração de um índice facetado como meio de tornar mais clara a análise da produção registrada em teses e dissertações na área de Comunicação. A sua proposta traz reflexões a respeito das possibilidades de transparência semântica dos modelos facetados. $O$ autor adota o método proposto pelo CRG e as categorias de Aristóteles como base metodológica de análise.

No contexto da produção de hiperdocumentos, Campos (2001) discute questões relativas à elaboração de sistemas voltados à organização do conhecimento, em especial os hiperdocumentos. Seu objetivo é propor critérios que fundamentem a elaboração de metodologias de construção de sistemas dessa natureza. Para tanto, utiliza como fundamentação teórica e metodológica a Teoria da Classificação, a Terminologia, o método Orientado a Objetos e a Ontologia, cujo elemento comum é o pensamento classificatório.

Esse tema está presente também em Campos e Gomes (2005). As autoras relatam ter o Método Facetado, concebido por Ranganathan, e a Teoria do Conceito, elaborada por Dahlberg, as bases teóricas e metodológicas essenciais para a construção de hiperdocumentos, respondendo às seguintes questões:

\begin{abstract}
Como "recortar" a temática a ser apresentada em unidades que possibilitem o entendimento do leitor: Como, no interior de uma unidade, deve-se relacionar os conceitos de forma a auxiliar o processo de compreensão do texto (CAMPOS; GOMES, 2005, p.6)
\end{abstract}

A primeira questão é respondida pelo Método Facetado, sendo a segunda resposta fundamentada na Teoria do Conceito.

Os hipertextos também são objeto de estudo de Lima (2004) em sua pesquisa de doutorado. Com base na problemática da inexistência de critérios semânticos durante a construção dos hipertextos e suas conseqüências na eficiência do processo de recuperação, a autora propõe um modelo hipertextual aplicado a teses e dissertações. Esse modelo toma por base as 
teorias da Análise Facetada e de Mapa Conceituais e resulta em um protótipo a ser implementado pela Biblioteca de Teses e Dissertações do Programa de PósGraduação da Escola de Ciência da Informação da UFMG.

No âmbito do desenvolvimento de sistemas, relatamos a ferramenta FASTAXON, elaborada por um grupo de especialistas em modelação conceitual. Esse instrumento, direcionado a elaboração de grandes taxonomias, baseia-se no paradigma da classificação facetada e na Álgebra de composição de termos compostos (Compound Term Composition Algebra). Tzitzikas et al. (2004) relatam que

\begin{abstract}
Ao usar o sistema, o elaborador define primeiramente o número de facetas e designa a cada uma delas uma taxonomia. Depois disso, o sistema pode gerar dinamicamente [...] uma navegação em árvore que permite ao elaborador (bem como ao indexador do objeto e ao usuário final) navegar os conjuntos de todos os possíveis termos compostos ${ }^{14}$ (tradução nossa).
\end{abstract}

A técnica da Álgebra de composição de termos compostos permite ao elaborador usar uma expressão algébrica para especificar os termos compostos válidos. É necessário apenas declarar um pequeno conjunto de termos compostos válidos ou inválidos, a partir do qual são inferidos outros termos compostos ( válidos e inválidos). Os autores finalizam o artigo relatando as tecnologias necessárias para implementação do FASTAXON.

Em um estudo anterior, Tzitzikas et al. (2002), apesar de não mencionar a ferramenta FASTAXON, descrevem um estudo semelhante, aplicado para catálogos na Web. Os autores relatam as vantagens das taxonomias facetadas em relação às hierárquicas. Segundo os autores, ser apto a inferir termos compostos em uma taxonomia facetada, impede erros, principalmente em casos de indexação feita por humanos. Além disso, a inferência de termos compostos possibilita a navegação em árvores por meio dos nós correspondentes aos termos válidos. Alertam, no entanto, que as taxonomias facetadas podem apresentar algumas desvantagens, pois permitem gerar um número muito grande de termos compostos inválidos. Apesar desse ponto negativo, aconselham o uso de taxonomias facetadas, pois estas auxiliam na clareza da estrutura conceitual. No intuito de resolver esse problema, criaram duas extensões de taxonomias facetadas denominadas PEFT (positive extended faceted taxonomies) para os termos compostos válidos e a NEFT (negative extended faceted taxonomies) para os termos compostos inválidos. O elaborador simplesmente declara os termos compostos válidos e/ou inválidos e esses são inferidos por um mecanismo baseado em implicações semânticas.

As taxonomias são também objeto de avaliação no artigo elaborado por Martinez et al. (2004). Nesse caso, são utilizadas as categorias fundamentais propostas pelo CRG para analisar taxonomias corporativas de alguns sites argentinos disponíveis na Web, cujos temas são: adega de vinho, indústria de alimentos, clube de futebol, associações profissionais, faculdades universitárias e agências governamentais. Os sites foram comparados com o intuito de determinar em que medida as categorias fundamentais do CRG estavam neles representadas. $\bigcirc$ estudo obteve o seguinte resultado: as adegas de vinho coincidiam em cerca de $81 \%$, as indústrias de alimentos em $68 \%$, os clubes de futebol em $43 \%$, as associações profissionais em $31 \%$, as faculdades em $50 \%$ e as agências do governo em (56\%). Com base nesse resultado, os autores concluem que, apesar de as categorias fundamentais do CRG não terem sido utilizadas deliberadamente pelos elaboradores dos sites, elas aparecem na sua estruturação, o que leva a considerá-las como um método válido a ser divulgado pelos bibliotecários e especialistas na área de elaboração de modelos baseados em facetas.

Straioto (2001) pesquisa a aplicabilidade do método facetado na análise de rótulos de xampu. $A$ autora inicia expondo as influências do trabalho de Ranganathan no contexto da organização do conhecimento, especificamente os estudos desenvolvidos pelo CRG, a elaboração do sistema PRECIS (Preserved Context Index System), o aparecimento dos tesauros facetados e o surgimento da ISKO (International Society for Knowledge Organization). Partindo dos principais

\footnotetext{
14 Using the system, the designer at first defines a number of facets and assigns to each one of them one taxonomy. After that the system can generate dynamically (and on the fly) a navigation tree that allows to the designer (as well to the object indexer or end user) to browse the set of all possible compound terms.
} 
aspectos teóricos-conceituais do método da construção de facetadas, Straioto analisa o caso da área de xampus, obtendo como resultado uma estrutura facetada para esse domínio. Essa pesquisa traz como elemento inovador a aplicação do método analíticosintético na representação de documentos técnicos.

Tristão et al. (2004) mostram a elaboração de uma classificação na área da cerâmica para revestimento. Os autores justificam a necessidade de um estudo dessa natureza relatando que "Embora exista normalização técnica sobre o assunto, as especificações apresentadas nas normas técnicas são desconhecidas e de difícil interpretação pela maioria dos usuários" (Tristão et al., 2004). No intuito de solucionar essa questão, o artigo apresenta um sistema de classificação para o setor, utilizando os métodos da classificação facetada, assim como as diretrizes da International Standard Organization referentes à classificação da informação na indústria da construção civil. Como resultado parcial, são apresentadas as facetas e os princípios de divisão adotados no sistema proposto. A determinação das facetas principais é estabelecida com base na análise dos campos semânticos Revestimento Cerâmico e Placas Cerâmicas.

Após uma exposição sobre formas de indexar informação na Web, como em Diretórios de assuntos e Mecanismos de busca, Ellis e Vasconcelos (2000) citam a aplicação desenvolvida por Duncan dos princípios da análise facetada para elaboração de base de conhecimento para construção de hipertextos. Os autores, ao relatar essa aplicação, citam ainda o uso desse estudo para elaboradores de materiais de ensino e como recurso para a geração de interfaces utilizando mapas conceituais baseados em tesauro. Os autores ressaltam os benefícios do uso da análise facetada no processo de busca na Web e organização de resultados como solução, mesmo que parcial, dos problemas relacionados à chamada indexação para usuário desconhecido, isto é, a falta de proximidade com aquele que busca a fonte, o que gera alto índice de recuperação irrelevante.

Guilten e Broad (2003) descrevem a reconstrução de um portal para o governo do estado voltado à informação sobre como iniciar um negócio. Os autores ressaltam a importância de conceitos clássicos da área de Ciência da Informação, como as facetas, que em nossos dias são essenciais na organização da arquitetura das informações de sites. $\bigcirc$ referido portal foi reorganizado com base no uso das facetas, objetivando oferecer uma visão mais lógica das partes em relação ao conteúdo global, o que o modelo antigo não conseguia atingir. São apontadas falhas no antigo sistema como a falta de consistência na criação das etiquetas, pouca flexibilidade no processo de navegação e carência de lógica na agregação de páginas, misturando elos entre tópicos de natureza diferentes. Inicialmente, foi elaborado um mapa de conteúdo baseado em um modelo informacional criado para organização dos tipos de informação contidos no site. Esse mapa foi aplicado no chamado Mental Model, que revelou inconsistências no conteúdo do portal, cuja resolução foi encontrada com o apoio do método facetado. A abordagem em facetas solucionou os problemas estruturais de etiquetagem, de navegação e do documento. $O$ produto resultante foi um portal de acesso mais intuitivo ao usuário. Ao final do documento, os autores indicam outros trabalhos de aplicação de facetas em áreas variadas da indústria.

Nesse contexto da arquitetura da informação, os autores Lovie, Maddox e Washington (2003) apresentam uma proposta de um sistema com modelagem facetada, utilizando tecnologias de software livre. De acordo com os autores, o método facetado é o mais adequado, pois consegue lidar com as mudanças constantes que ocorrem na representação do conhecimento humano real. $\bigcirc$ protótipo gerencia o sistema de classificação facetada, trata os documentos utilizando o esquema facetado e publica-os em um site cujo mapa é construído com base na classificação.

$\mathrm{Na}$ linha do tratamento do conteúdo de materiais audiovisuais, citamos o artigo de Del Valle Gastaminza et al. (2002) que propõe a organização de um tesauro para a rede de televisão Telecinco com a finalidade de integrar todo o documento em um meio digital inovador. Esse tesauro usa como fundamento metodológico os princípios das linguagens epistemográficas que, segundo o autor, "representam um intuito de solucionar tanto a inflexibilidade das linguagens documentárias tradicionais como sua falta de adequação ao campo da informação de atualidade e a área de materiais audiovisuais"15 (Del Valle Gastaminza et al., 2002, p. 108) (tradução nossa). Essas

\footnotetext{
15 [...] que representan un intento de solucionar tanto el anquilosamiento de los lenguajes documentales tradicionales como su falta de adecuación al campo de la información de actualidad y al dominio de los materiales audiovisuales.
} 
linguagens de estrutura associativa resultam de construções cognitivas que funcionam como uma base de conhecimento cujos objetivos são a organização documentária e a oferta de alternativas de recuperação da informação. Uma das grandes contribuições dessas linguagens deriva de

[...] sua capacidade para criar representações cognitivas derivadas de desenvolvimento lógico-semântico e discursivos, que têm como objetivo esboçar representações pragmáticas (referentes ao uso) de mapas cognitivos, simulando enunciados do discurso mediante a macroestrutura e a microestrutura"16 (Del Valle Gastaminza et al., 2002, p. 108) (tradução nossa).

A abordagem facetada é aplicada na definição das macrocategorias. Os autores fundamentam essa etapa nos estudos do Art \& Architecture Thesaurus, García Gutiérrez, Ranganathan e van Slype.

No âmbito dos sistemas de recuperação da informação em linha, Pollitt (1998) mostra o valor cada vez maior das classificações e tesauros como ferramentas de aprimoramento tanto das buscas como das formas de apresentação dos resultados de uma pesquisa. $\bigcirc$ autor descreve o sistema HIBROWSE (High resolution Interface for BROWing and Searching), baseado na abordagem denominada pelo autor de View-based searching. Pollitt (1998), ressalta que essa abordagem segue as recomendações de Ranganathan e do CRG, aplicando as potenciais possibilidades de utilização de tesauro facetado nos dois momentos do tratamento da informação: busca e resultados. Uma descrição da aplicação do HIBOWSE na base de dados em literatura médica EMBASE, utilizando o tesauro EMTREE comprova a eficiência da abordagem. O autor ainda discute a relevância desse enfoque para as OPACs (Online public access catalog).

Pollitt, Smith e Braekevelt (1998), em outro artigo, desenvolvem mais profundamente o sistema HIBROWSE, enfocando principalmente a interface de recuperação da informação baseada na classificação facetada. Segundo os autores, por meio do uso automático de operadores Booleanos, o sistema filtra as pesquisas com base nas escolhas de facetas e atributos realizadas pelo usuário.

É o tema também de investigações sobre o potencial dos tesauros facetados na recuperação da informação, o Projeto FACET coordenado por Douglas Tudhope da Universidade de Glamorgan (Universidade..., 2007), na Inglaterra. Esse estudo tem por objetivo pesquisar o uso de tesauros facetados em técnicas e interfaces de busca, sem que o usuário tenha que necessariamente utilizar exatamente a forma como foi realizada a indexação. Para tanto, aplica medidas de aproximação semântica com base nos relacionamentos do tesauro e no contexto das facetas para guiar, de forma automática e interativa, a expansão ou refinamento dos termos do tesauro durante a realização das buscas. Adota igualmente essas medidas na apresentação dos resultados das buscas efetuadas.

Voltada para o formalismo adotado em sistemas de conhecimento, Priss (2000b) defende ser a representação do conhecimento facetado a que melhor se adapta a esse tipo de implementação. Com o intuito de propor uma teoria formal de facetas com aplicação em sistemas de conhecimento facetado, o artigo apresenta primeiramente as noções básicas da representação desse tipo de conhecimento. Em seguida, o formalismo é modelado em um tesauro facetado.

Em outro artigo, Priss (1998) descreve uma interface gráfica para navegação e construção de tesauro facetado, fundamentada na chamada análise conceitual formal. A aplicação da formalização matemática garante a consistência das relações semânticas enquanto a interface gráfica, implementada em Java, orienta o usuário na navegação entre termos e facetas. Clicando em um termo o usuário tem acesso às facetas e componentes.

A análise conceitual formal descrita por Priss (2000a) é aplicada em outro estudo cujo objetivo é comparar qualitativamente diferentes sistemas utilizando facetas. Para tanto, Priss adota como corpus de análise a Classificação Decimal de Dewey e o Yahoo.

Nesse estudo, Priss (1999) apresenta uma descrição lógica da representação facetada do conhecimento com vistas a facilitar traduções entre diferentes formalismos de representação do

\footnotetext{
16 [...] su capacidad para crear representaciones cognitivas derivadas de desarrollos lógico-semánticos y discursivos que tienen como objetivo plantear representaciones pragmáticas (referidas al uso) de mapas cognitivos, simulando enunciados del discurso mediante la macroestrutura y la microestrutura".
} 
conhecimento. A autora propõe como aplicações possíveis a construção de tesauros facetados e a mineração de dados.

Fox (2005) explora as possibilidades de aplicação da Teoria da Classificação Facetada de Ranganathan nas questões de acessibilidade e exposição de conteúdos de repositórios digitais. $\bigcirc$ autor expõe a necessidade atual das ciências em serem classificadas por um sistema cujo vocabulário reflita os padrões de cada área do conhecimento. Essa problemática é sentida principalmente hoje com a rápida proliferação dos repositórios digitais e a necessidade crescente de esquemas de classificação mais ágeis e flexíveis que possam ser implementados na moderna tecnologia atual. Esse tipo de flexibilidade é possível quando se adota uma abordagem como a desenvolvida por Ranganathan, uma vez que ela determina vários pontos de acesso à informação pelo uso do método facetado. Fox ressalta que a metodologia facetada pode ajudar bibliotecas a exporem seus enormes repositórios de conteúdos digitais dentro de um sistema muito flexível, o que levará ao aprimoramento dos estudos em Web semântica. Nesse contexto, o autor cita o sistema de gerenciamento digital institucional Dspace (http:// www.dspace.org) e os benefícios da abordagem facetada na elaboração de classificações que descrevam objetos digitais. Segundo o autor, a Classificação Facetada de Ranganathan é um excelente modelo a ser aplicado na classificação de conteúdos digitais, apoiando a interface dos arquivos digitais com uma Web semântica.

Broughton (2002) apresenta uma aplicação da Teoria da Classificação Facetada nos portais Arts and Humanities Data service, e Humbul. Trata-se de um projeto conjunto entre School of Library, Archive and Information Studies da University College London e a

\section{REFER Ê NCIAS}

ARAÚJO, C.A.A. Contribuições da classificação facetada no mapeamento do campo da comunicação. In: CONGRESSO BRASILEIRO DE CIÊNCIAS DA COMUNICAÇÃO, 26., 2003, Belo Horizonte. Anais... Belo Horizonte, 2003.

BARDIN, L. L'analyse du contenu. 7.éd. Paris: PUF, 2003. 296p. (Le Psychologue, 69).

BROUGHTON, V. Facet analytical theory as a basis for a knowledge organization tool in a subject portal. In: LÓPEZ-HUERTAS, María J. (Ed.). Challenges in knowledge representation and organization for the 21 st century: integration of knowledge across boundaries: proceedings of the Seventh International ISKO Conference, 2002,
United Kingdom Higher Education. $O$ estudo mostra a utilidade dos esquemas classificatórios do tipo facetado na organização e tratamento do conteúdo de recursos digitais. A autora aponta a estrutura facetada como a forma mais viável para o gerenciamento de terminologias e conceitos em um meio diversificado como o portal de Artes. Relata igualmente ser esse tipo de classificação o mais adequado para o desenvolvimento de ferramentas para o gerenciamento do vocabulário, descrição de documentos e sua recuperação. Do ponto de vista metodológico, são aplicados os princípios classificatórios do CRG e adotada a Classificação Bibliográfica de Bliss (segunda edição), como padrão de organização do conteúdo.

\section{O NCLUSÃO}

registro de pesquisas sobre reflexões teóricas e aplicações levantadas por esta pesquisa mostra a importância e a atualidade dos instrumentos que seguem o princípio analítico-sintético. As questões teóricas refletem preocupações quanto à clareza dos conceitos sobre facetas. No intuito de tornar essas noções mais precisas, alguns autores propõem sínteses, outros utilizam o método comparativo ou da análise da utilização desses conceitos em documentos normativos e metodologias de construção de modelos facetados. No que concerne às aplicações, são de natureza múltipla e atual, o que vem comprovar a adaptabilidade do método.

A apresentação do conteúdo dos artigos identificados na literatura foi o intuito específico deste estudo, concluindo a primeira parte da pesquisa. $\bigcirc$ levantamento realizado foi necessário para verificar as possibilidades de aplicação do modelo de predicação sintático-semântico proposto por Café (1999), objeto da segunda parte do estudo ainda a ser realizado.

Granada. Würzburg: Ergon Verlag, 2002. Disponível em: <http:/ /www.ucl.ac.uk/fatks/paper2.htm>. Acesso em: 26 jun. 2006.

CAFÉ, L. La description et l'analyse des unités terminologiques complexes en langue portugaise (variété brésilienne): une contribution à l'automatisation de la banque de données terminologiques du Brésil (Brasilterm). 1999. 2v. Tese (Doutorado em Lingǘstica) - Département de Langues et Linguistique, Université Laval.

CAMPOS, M.L.A. Modelização de domínios de conhecimento: uma investigação de princípios fundamentais. Ciência da Informação, v.33, n. 1, p.22-32, 2004. 
CAMPOS, M.L.A. A organização de unidades do conhecimento em hiperdocumentos: o modelo conceitual como um espaço comunicacional para realização da autoria. 2001. 198f. Tese (Doutorado em Ciência da Informação) - Universidade Federal do Rio de Janeiro.

CAMPOS, M.L.A.; GOMES, H.E. Princípios de organização e representação do conhecimento na construção de hiperdocumentos. DataGramaZero, v.6, n.6, p. 1-12, 2005. Disponível em: <http://datagramazero.org.br/dez05/Arti_04.htm>. Acesso em: 25 jan. 2006.

CLASSIFICATION RESEARCH GROUP. The need for a faceted classification as the basis of all methods of information retrieval. In: CHAN, L.M.; RICHMOND, P.A.; SVENONIUS, E. (Ed.) The theory of subject analysis: a sourcebook. Colorado, Libraries Unlimited, 1985, p.154-167.

DAHLBERG, I. Teoria do conceito. Ciência da Informação, v.7, n.2, p. 101-107, 1978

DEL VALLE GASTAMINZA, F. et al. Construcción de un tesauro para el Centro de Documentación del Telecinco. Scire: Representación y Organización del Conocimiento, v.8, n. 1, p.103-118, 2002. Número monográfico: Perspectivas en representación del conocimiento.

ELLIS, D.; VASCONCELOS, A. The relevance of facet analysis for World Wide Web subject organization and searchig. Journal of Internet Cataloging, v.2, n.3, p.97-114, 2000.

FOX, R. Cataloging our information architecture. OCLC Systems \& Services: International Digital Library Perspectives, v.21, n. 1, 2005. p.23-29. Disponível em: <http:// www.ingentaconnect.com/content/mcb/164/2005/00000021/ 00000001/art00005 > . Acesso em: 28 jun.2006 .

GLASSEL, A. Was Ranganathan a Yahoo!? InterNICNews, Internet Scout Projet, March 1998. Disponível em: <http://scout.wisc.edu/ Projects/PastProjects/toolkit/enduser/archive/1998/euc9803.html>. Acesso em: 27 jun. 2006.

GNOLI, C. Is there a role for tradicional knowledge organization system in the digital age? E-IIS: E-prints in Library and Information Science, v.1, n.1, p.1-6, 2004. Disponível em: <http:// eprints.rclis.org/archive/00001415/fullmetadata.html>. Acesso em: 16 fev. 2005.

GUILTEN, B.; BROAD, J. Faceted approach to Web Redesign. In: IAWIKI: the wiki for information architecture, 2003. Disponível em: <http://www.iawiki.net/FacetedApproach>. Acesso em 8 jul. 2006.

HJØRLAND, B. The methodology of constructing classification schemes: a discussion of the state-of-the-art. In: INTERNATIONAL ISKO CONFERENCE, 7., 2002, Granada. Proceedings... Würzburg: Ergon Verlag, 2002. p. 450-456. (Advances in Knowledge Organization, 8)

KWASNIK, B.H. The role of classification in knowledge representation and discovery (1). Library Trends, v.48, p. 1-22,
1999. Disponível em: <http://infotrac.galegroup.com/itw/ infomark/463/409/78669675w4/p... >. Acesso em: 27 jan. 2006.

LEISE, F. Using faceted classification to assist indexing. In:--

Contextual analysis. Chicago, c2003. p. 1-5. Disponível em: <http:/ /www.contextualanalysis.com/pub_usingfacets.php > . Acesso em: 28 jun. 2006.

LIMA, G.A.B. A análise facetada na modelagem conceitual de sistemas de hipertexto: uma revisão de literatura. Perspectivas em Ciência da Informação, v.7, n.2, p. 189-196, 2002.

LIMA, G.A.B.O. Mapa hipertextual (MHTX) um modelo para a organização hipertextual de documentos. 2004. 199f. Tese (Doutorado em Ciência da Informação) - Escola de Ciência da Informação, Universidade Federal de Minas Gerais. Disponível em: <http://www.gercinalima.com/glima/modules/wfchannel/ index.php?pagenum =11>. Acesso em: 12 jul. 2007.

LOUIE, A.J.; MADDOX, E.L; WASHINGTON, W. Using faceted classification to provide structure for information architecture. In: INFORMATION ARCHITECTURE SUMMIT OF THE AMERICAN SOCIETY FOR INFORMATION SCIENCE AND TECHNOLOGY, 2003, Portland, University of Washington Program for Educational Transformation Through Technology. Disponível em: <http:// depts.washington.edu/pettt/presentations/conf_2003/ IASummit.pdf>. Acesso em: 28 jun. 2006. Pôster.

MANIEZ, J. Des classification aux thesaurus: du bon usage des facettes. Documentaliste, v.36, n.4-5, p.249-262, 1999.

MAPLE, A. Faceted access: a review of the literature. 1995. Disponível em: <http://www.music.indiana.edu/tech s/mla/ facacc.rev> . Acesso em: 28 jun. 2006.

MARTINEZ, A. et al. Las categorias o facetas fundamentales: una metodologia para el diseño de taxonomias corporativas de sitios Web argentinos. Ciência da Informação, v.33, n.2, p.106-1 11 , 2004.

MILLS, J. Faceted classification and logical division in information retrieval. Library Trends, v.52, n.3, p.541-70, 2004. Disponível em: <http://vnweb.hwwilsonweb.com/hww/shared/ shared main.jhtml? requestid $=13486>$. Acesso em: 28 jun. 2006.

PEREIRA, E.C.; BUFREM, L.S. Princípios de organização e representação de conceitos em linguagens documentárias. Encontros BIBLI: Revista Eletrônica de Biblioteconomia e Ciência da Informação, n.20, p.21-37, $2^{\circ}$ semestre 2005.

POLLITT, A.S. The key role of classification and indexing in viewbased searching. International Cataloguing and Bibliographic Control, v.27, n.2, p.1-8, 1998. Disponível em: <http:// www.ifla.org/IV/ifla63/63polst.pdf>. Acesso em: 28 jun. 2006.

POLLITT, A.S.; SMITH, M.P.; BRAEKEVELT, P.A.J. View-based searching systems: new paradigm for information retrieval based on faceted classification and indexing using mutually constraining knowledge-based views, In: OCLC DISTINGUISHED SEMINAR SERIES, 1998, Dublin. Disponível em: <http://www.view-basedsystems.com/papers/bcshci.htm >. Acesso em: 28 jun. 2006. 
PRISS, U. Comparing classification systems using facets. In: INTERNATIONAL ISKO CONFERENCE, 6., 2000. Proceedings... 2000a. p.170-175. (Advances in Knowledge Organization, 7)

PRISS, U. Description logic and faceted knowledge representation. CEUR Workshop Procedings, v. 22, 1999.

PRISS, U. Faceted knowledge representation. Electronic Transaction on Artificial Intelligence, v. 4, p.21-33, 2000b. Disponível em: <http://www.ep.liu.se/ej/etai/2000/002>. Acesso em: 26 jun. 2006.

PRISS, U. A Graphical interface for conceptually navigating faceted thesauri. In: INTERNATIONAL ISKO CONFERENCE, 5., 1998. Procedings... 1998. p.184-190. (Advances in Knowledge Organization, 6)

RANGANATHAN, S.R. Facet analysis: fundamental categories. In: CHAN, L.M.; RICHMOND, P.A.; SVENONIUS, E. (Ed.). Theory of subject analysis: a sourcebook. Colorado, Libraries Unlimited, 1985. p.86-93.

SOERGEL, D. The rise of ontologies or the reinventation of classification. Journal of the American Society for Information Science, v.50, n. 12, p. $1119-1120,1999$.

SPITERI, L.F. The essential elements of faceted thesauri. Cataloging \& Classification Quarterly, v. 28, n. 4, p.31-52, 1999.

SPITERI, L.F. A simplified model for facet analysis. Canadian Journal of Information and Library Science, v. 23, p. 1-33, 1998.
SPITERI, L.F. The use of facet analysis in information retrieval thesauri: an examination of selected guidelines for thesaurus construction. Cataloging \& Classification Quarterly, v.25, p.21-38, 1997.

STRAIOTO, A.C. A análise facetada como dimensão teórica e prática na organização do conhecimento. 2001. 163f. Dissertação (Mestrado em Ciência da Informação) - Faculdade de Filosofia e Ciências, Universidade Estadual Paulista.

TRISTÃO, A.M.D., FACHIN, G.R.B., ALARCON, O.E. Sistema de classificação facetada e tesauro: instrumentos para organização do conhecimento. Ciência da Informação, v.33, n.2, p.161-171, 2004

TRISTÃO, A.M.D. et al. Sistema de classificação facetada: instrumento para organização da informação sobre cerâmica para revestimento. Informação \& Sociedade: Estudos, v. 14, n.2, p. 1 21, 2004. Disponível em:

<http://www.informacaoesociedade.ufpb.br/ojs2/index.php/ies/ article/viewFile/62/60> . Acesso em: 26 jun. 2006.

TZITZIKAS, Y. et al. Extended faceted taxonomies for Web Catalog. ERCIM News, n.51, p.1-2, 2002. Disponível em: <http:// www.ercim.org/publication/Ercim News/enw51/tzitzikas.html> Acesso em: 26 jun. 2006.

TZITZIKAS, Y. et al. Fastaxon: a system for fast (and faceted) taxonomy design. In: INTENATIONAL CONFERENCE ON CONCEPTUAL MODELING, 23. , 2004, Sanghai. Anais... Sanghai, 2004.

UNIVERSITY OF GLAMORGAN. The Facet Projet. 2007. Disponível em: <http://www.comp.glam.ac.uk/\%7EFACET/>. Acesso em: 13 jul. 2007. 